\title{
June 2020 Pulmonary Case of the Month: Twist and Shout
}

\author{
Lewis J. Wesselius, $\mathrm{MD}^{1}$ \\ Staci E. Beamer, MD2 \\ ${ }^{1}$ Departments of Pulmonary Medicine and ${ }^{2}$ Thoracic Surgery \\ Mayo Clinic Arizona \\ Scottsdale, AZ USA
}

\section{History of Present IIIness}

An 83-year-old man presented with a left upper lobe lung nodule. The nodule was noted on a routine follow-up chest radiograph obtained after a radical cystectomy and left nephro-ureterectomy done 9 months earlier for invasive bladder cancer as well clear cell carcinoma of left kidney. He had symptoms of a mild chronic cough but denied shortness of breath with activities of daily living.

\section{$\mathrm{PMH}, \mathrm{SH}, \mathrm{FH}$}

- Prostate cancer, post prostatectomy in 2009.

- Bladder cancer and left renal cell cancer resected in Jan 2019

- Post-op chemotherapy after bladder and left kidney resections

- Non-ischemic cardiomyopathy, possibly due to chemotherapy, EF $45 \%$

- Chronic atrial fibrillation

- Smoking history: 60 pack years, no occupational exposures

\section{Physical Examination}

Other than an irregular pulse, his physical examination was unremarkable.

\section{Medications}

- Warfarin

- Atorvastatin

- Hydrochlorothiazide

- Ramipril

- Atenolol

\section{Radiography}

The initial chest radiograph is shown in Figure 1. 


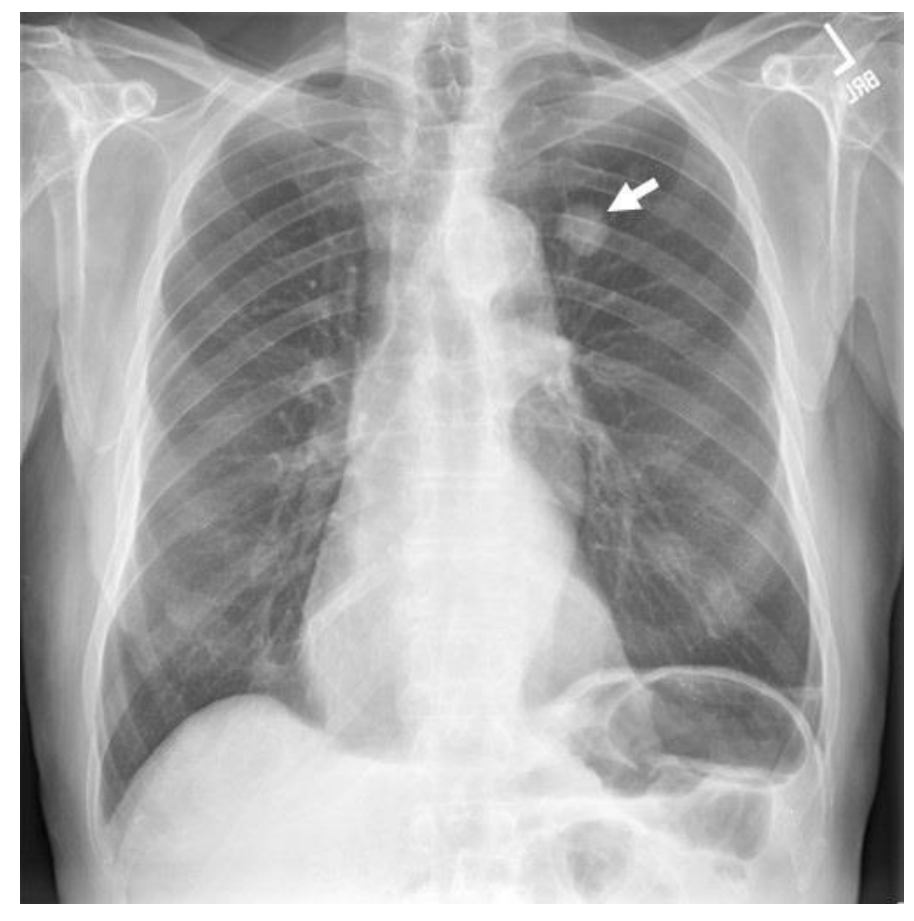

Figure 1. Initial chest x-ray.

Which of the following should be done at this time?

1. Chest CT scan

2. Left upper lobectomy

3. Comparison to old chest $x$-rays

4. 1 and 3

5. All of the above 


\section{Correct!}

\section{1 and 3}

Comparison to a previous chest $x$-ray is a good idea although it is unlikely the $2.7 \mathrm{~cm}$ nodule would be missed. Previous chest-x-rays did not show the nodule. A thoracic CT scan will confirm the presence of the nodule as well as ensuring that other pathology is not present such as other nodules of mediastinal lymphadenopathy. Since there are a number of possibilities for the nodule including metastatic bladder or kidney cancer, primary lung cancer or an infection such as coccioidomycosis, a thoracic CT scan may help narrow the differential. The thoracic CT scan showed only the presence of the left upper lobe nodule (Figure 2).

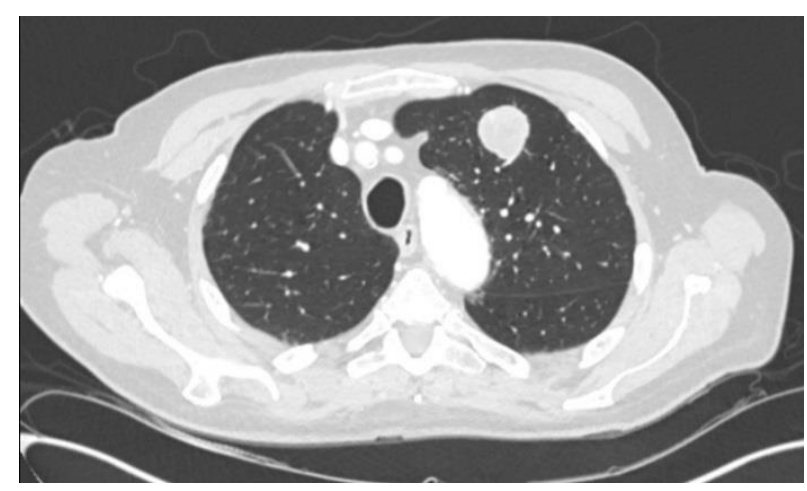

Figure 2. Representative image from thoracic CT scan showing left upper lobe nodule without calcium. No other nodules or lymphadenopathy was identified.

What should be done at this time?

1. Bronchoscopy

2. Left upper lobectomy

3. Needle biopsy of lung

4. 1 or 3

5. Any of the above 


\section{Correct! \\ 3. Needle biopsy of lung}

Bronchoscopy is not necessarily a wrong choice but the nodule is peripheral and a needle biopsy would have a higher diagnostic yield. Left upper lobectomy without knowing the cause of the nodule may be wrong. A CT guided needle biopsy was performed. The histopathology was consistent with a metastatic urothelial tumor.

Which of the following should be done at this time?

1. Cisplatinum-based chemotherapy

2. Left pneumonectomy

3. Left upper lobectomy

4. Oncology consult

5. Radiation therapy 


\section{Correct! \\ 4. Oncology consult}

Owing to the rarity of metastatic uroepithelial carcinoma, there are insufficient data to provide strong recommendations (1). However, the results of recent multicentre studies are now available and cisplatinum-based chemotherapy is usually recommended. There are also data suggesting that resection of a solitary pulmonary metastasis may improve survival. However, these are judgement calls and oncology consultation would usually be advised. Oncology did recommend left upper lobectomy followed by chemotherapy. Thoracic surgery was consulted and the patient was thought to be a good lobectomy candidate. A left upper lobe robotic lobectomy performed without evidence of complication.

A postoperative chest $x$-ray is shown in Figure 3.

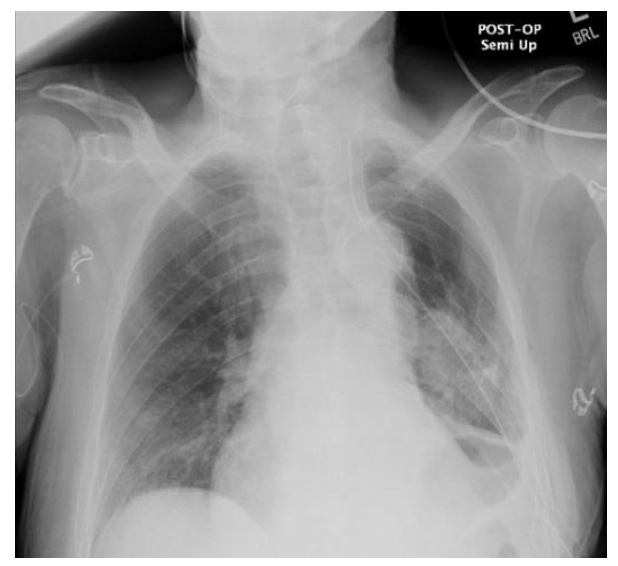

Figure 3. Post-operative portable chest x-ray showing some increased left basal consolidation and small apical pneumothorax.

Overnight, the patient became hypotensive (BP 90/60).

What should be done at this time?

1. Give a bolus of normal saline followed by an increase in IV fluids

2. Repeat the portable chest $\mathrm{x}$-ray

3. Transfer the patient to the ICU

4. 1 and 3

5. All of the above 


\section{Correct!}

\section{All of the above}

It is not entirely clear why the patient became hypotensive and a transfer to the ICU for monitoring seems reasonable. He initially responded to a bolus of IV fluids and a repeat chest portable chest $x$-ray is shown in Figure 4.

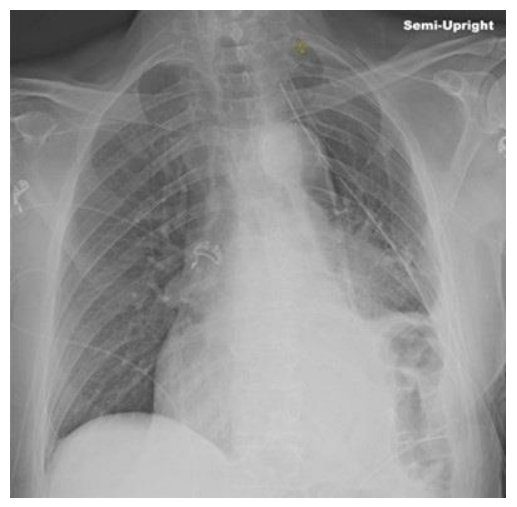

Figure 4. Repeat post-operative portable chest x-ray again showing the left basal consolidation which is not markedly different compared to the previous chest $\mathrm{x}$-ray (Figure 3).

However, the following morning the patient developed increasing dyspnea and hypotension. He was re-intubated and started on dobutamine and norepinephrine. Another portable chest $\mathrm{x}$-ray was performed (Figure 5).

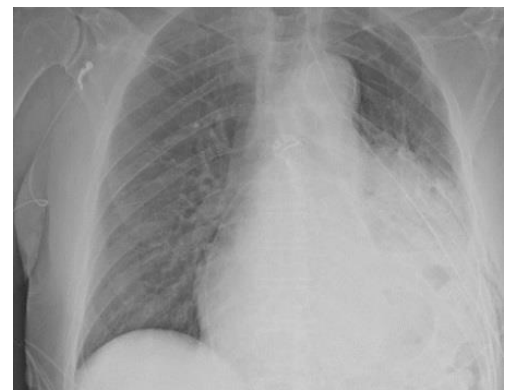

Figure 5. A third post-operative portable chest x-ray again showing an increase in the left basal consolidation.

What should be done at this time?

1. Bronchoscopy

2. Insert a second chest tube for increased pneumothorax

3. Thoracic CT scan

4. 1 and 3

5. All of the above 


\section{Correct!}

\section{1 and 3}

The chest x-ray does not reveal a large pneumothorax. Representative images of the thoracic CT scan are shown in Figure 6.

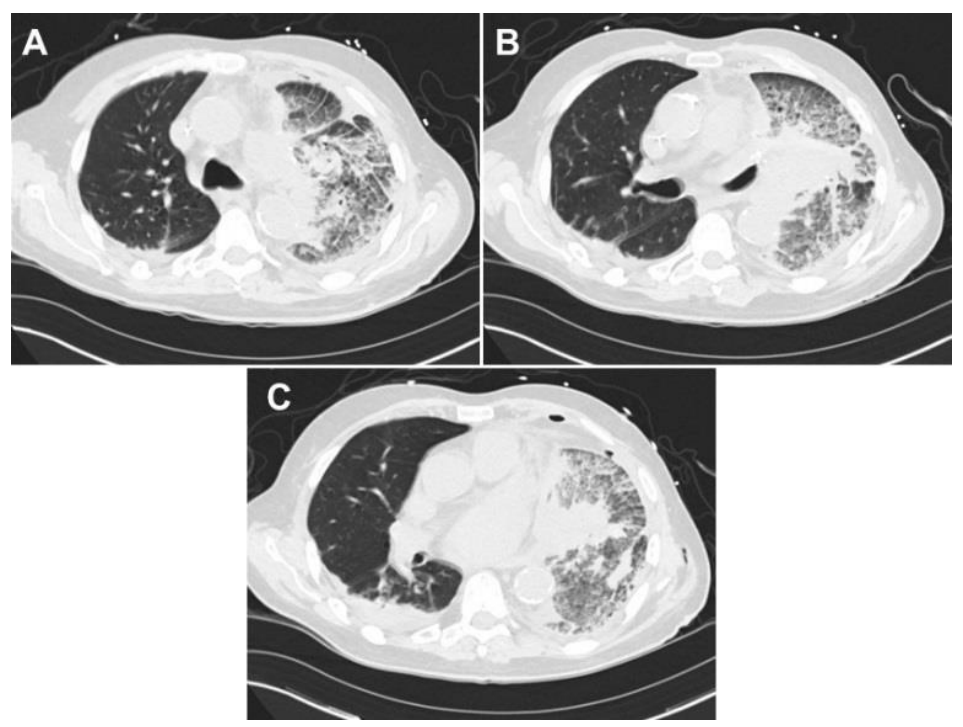

Figure 6. Representative images from the thoracic CT scan in lung windows showing obstruction of the left lower lobe bronchus and a mass-like consolidation in left peri-hilar region with surrounding ground-glass and septal thickening in entire remaining left lung.

Bronchoscopy showed the left lower lobe bronchus to be narrowed (Figure 7).

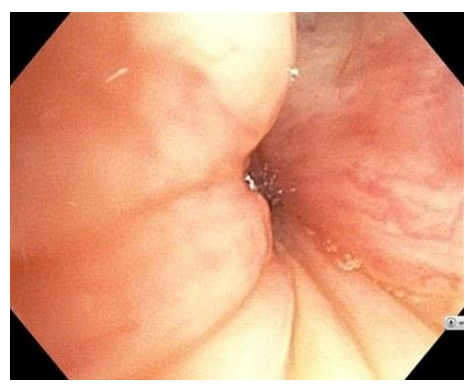

Figure 7. Bronchoscopic photograph showing narrowing of the left lower lobe bronchus.

What should be done next?

1. Administer broad spectrum antibiotics for hospital acquired pneumonia

2. Anticoagulation for pulmonary embolism

3. Diuresis for pulmonary edema

4. Insert a counterpulsating balloon pump for aortic dissection

5. Take patient to the operating room for lobar torsion 


\section{Correct! \\ 5. Take patient to the operating room for lobar torsion}

The patient has lobar torsion of his left lower lobe. There is no evidence of infectious pneumonia, pulmonary embolism, pulmonary edema, or aortic dissection, the later a contraindication to a counterpulsating balloon pump. The patient was taken back to the operating room. Video-assisted thoracoscopic surgery (VATS) demonstrated diffuse hemorrhage in the remaining left lung which appeared non-viable. The patient underwent completion pneumonectomy performed via thoracotomy.

Which of the following are true regarding lobar torsion?

1. Lobar torsion is often fatal unless quickly recognized

2. Lobar torsion is rare

3. Most commonly occurs in the right middle lobe following right upper lobectomy

4. 1 and 3

5. All of the above 


\section{Correct!}

\section{All of the above}

Lobar torsion is a rare but potentially fatal post-lobectomy complication (2). It most commonly involves the right middle lobe after a right upper or right lower lobectomy, but also occurs in left lung. The incidence is variably reported as $0.089 \%$ to $0.3 \%$. The pathophysiology is controversial. Lobar torsion occurs due to an axial, usually clockwise, rotation of the lung with subsequent airway and vascular obstruction. An attempt at surgical de-torsion can be considered if the lung appears viable, but in the majority of cases a completion pneumonectomy is life-saving as these patients can deteriorate quickly as lung develops gangrene.

The patient recovered well postoperatively and has done well in follow-up.

\section{References}

1. Rouprêt $\mathrm{M}$, Babjuk $\mathrm{M}$, Compérat $\mathrm{E}$, et al. European association of urology guidelines on upper urinary tract urothelial carcinoma: 2017 update. Eur Urol. 2018;73(1):11122. [CrossRef] [PubMed]

2. Childs $L$, Ellis $S$, Francies $O$. Pulmonary lobar torsion: a rare complication following pulmonary resection, but one not to miss. BJR Case Rep. 2017; 3(1): 20160010. [CrossRef] [PubMed] 